\section{A Transatlantic Communication Experiment via Echo I Satellite}

The article in Nature of January 28, p. 268, by Carru, Gendrin and Reyssat, described the reception by the Centre National d'Etude des Télécommunications of $960-\mathrm{Mc}$./s. signals reflected from the Echo I satellite during pass 70 .

The signals originated at the Bell Telephone Laboratories' station in Holmdel, New Jersey, U.S.A. The transmitter frequency was measured just before pass 70 and found to be $960.045 \mathrm{Mc}$./s., which is in good agreement with the value of $960 \cdot 045053 \mathrm{Mc} / \mathrm{s}$. calculated by Carru et al. during reception some 20 min. later.

The 60-ft. diameter transmitting antenna used at Holmdel has a gain of $43 \cdot 1+0 \cdot 1 \mathrm{db}$. instead of the value of $44 \mathrm{db}$. assumed by Carru et al. Transmitter power at the time of reception was $7.5 \mathrm{~kW}$. and was further reduced by the $0.5 \mathrm{db}$. antenna-transmission line loss to $6.7 \mathrm{~kW}$., which is $0.8 \mathrm{db}$. less than that assumed by Carru et al. Inspection of pointing records for the reception period shows that the Holmdel antenna was aimed to within $0 \cdot 2^{\circ}$ of the satellite, which would add an additional loss of no more than $0 \cdot 1 \mathrm{db}$. These factors predict a smaller value for received signal by $1.8 \mathrm{db}$.; on the other hand, there is no justification for the assumption that the cross-section of the balloon varies with the geometry of the transmission path, as borne out by our own measurements on Echo $I$ and the correct theoretical treatment ${ }^{1}$. Thus the $1 \cdot 3 \mathrm{db}$. factor used by Carru et al. can be omitted, leaving a net loss of $0.5 \mathrm{db}$., or a predicted received level of $-143.0 \mathrm{dbm}$. This is in good agreement with the value reported, $-142 \mathrm{dbm}$. $\pm 1 \mathrm{db}$.

Accurate information regarding the Holmdel transmission is available on request to any independent experimenters who need such information to evaluate their own measurements.

The results of the radio transmission tests supported by the U.S. National Aeronautics and Space Administration and using the Echo I satellite over the period August 1960-March 1961 have been evaluated and will be published shortly.

WM. C. JAKES, JUN.

Bell Telephone Laboratories, Inc. Holmdel,

New Jersey.

1 Morgan, et al., Proc. Inst. Rad. Eng., 48, 1781 (October 1960).

WE thank Mr. Jakes for the above information, which corroborates the order of magnitude of our measurements and assumptions. The reference which he points out had only just been published when we submitted our communication (we added it to our ref. 4) and we had not had time to investigate the case of circular polarization, which is not thoroughly examined in Morgan's paper.

H. Carru

R. GENDRIN

M. Reyssat

Centre National D'Etudes des Télécommunications, 3 avenue de la République,

Issy-les-Moulineaux (Seine)

\section{PHYSICS}

\section{Apparent Intermediate Supervelocity of Detonation}

The transition from the low to the high detonation velocity in a cylindrical dynamite charge is seen as a rather sharp bend in the streak camera curve. At the transition point the high velocity often starts with an apparent supervelocity which is much greater than the stationary high velocity. The reason is probably that the high velocity is initiated by the low velocity at a point on the axis. Good accordance between observed and calculated values is obtained if it is assumed that the high-velocity detonation sets off from a point a little behind the luminous zone of the low velocity. This accords with the fact that the latter, according to instantaneous photographs, is at the front of the preliminary reaction zone of Selberg and Sjölin ${ }^{2}$.

An analogous phenomenon is the initiation of a cylindrical charge by an axial shock-wave. In that case the detonation sets out from a circular, coaxial area on the shock front, the radius of which is smaller than the radius of the charge ${ }^{2}$, and the maximum apparent supervelocity goes to infinity.

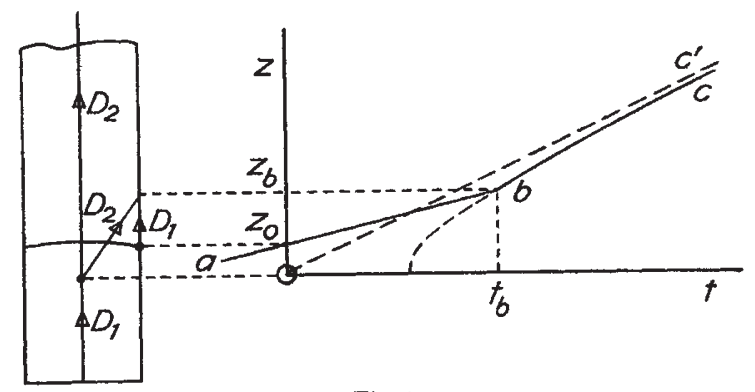

Fig. 1

Fig. 1 gives a schematic picture of the course of events. To the left is shown the cylindrical charge with the front of the low velocity and the starting point of the high velocity when the high velocity starts $(t=0)$. In the time diagram to the right, $a b c$ shows the course of the luminous front at the envelope surface of the charge. It is obtained from the streak camera photography. $b$ is the point where the high-velocity front overtakes the low-velocity front. $O c^{\prime}$ shows the front of the high-velocity detonation along the axis. The slope of $a b$ gives the low velocity $D_{1}$, and the derivative of the curve

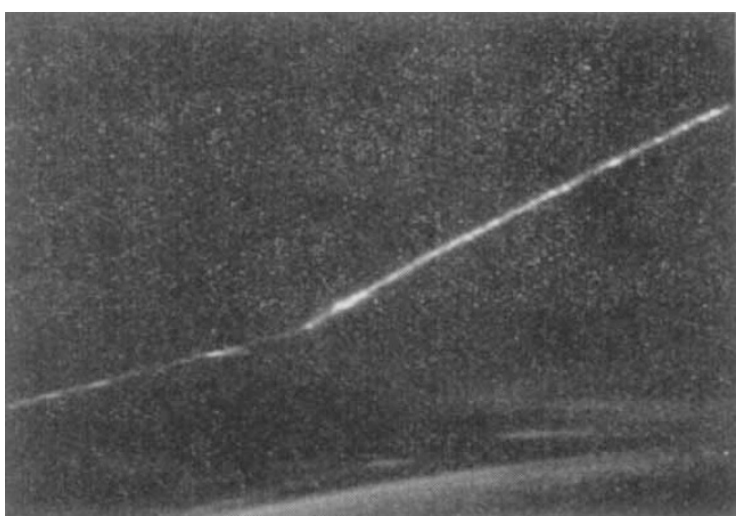

Fig. 2 